\title{
Experimental Evidences of Redistribution Phenomenon in Supercapacitors and Consequent Improvement of their Dynamic Model
}

\author{
Dimitri Torregrossa, Maryam Bahramipanah, Rachid Cherkaoui, Mario Paolone \\ Distributed Electric System Laboratory \\ Swiss Federal Institute of Lausanne EPFL Lausanne, Switzerland
}

\begin{abstract}
The paper presents a set of experimental investigations related to the study of the dynamic behavior of supercapacitors. The experimentally-inferred results are then used as inputs for an improved version of one of the most common supercapacitor RC-circuit models. The key improvement concerns the accurate modeling of the redistribution phenomenon of the supercapacitor residual charge during charging/discharging and relaxation phases. The accuracy of the obtained model has been experimentally validated. Preliminary physical investigations concerning the temperature effects on the SC dynamic behavior are also presented and discussed.
\end{abstract}

Index Terms-Supercapacitor modeling, redistribution phenomenon, residual charge, dynamic supercapacitors behavior, temperature effects.

\section{INTRODUCTION}

Supercapacitors (SCs), also known as ultracapacitors, are electric devices used in a large spectrum of energy-storage applications that require fast cycles characterized by high power flows. Typical examples are: fast charging of electric vehicles, medical devices, universal power supply (UPS), elevators, pulsed laser, etc.

As known, SCs are characterized by long life cycles (even more than one million of cycles), high power density (5-7 $\mathrm{kW} / \mathrm{kg}$ ), low energy densities (in the order of few tens of $\mathrm{Wh} / \mathrm{kg}$ ) and short charging times.

The research activity about the development and modeling of these devices has increased during the last ten years and several papers concerning modeling [1-10] and ageing [11-12] of these energy storage systems have been published. References [1-9] have proposed the representation of the dynamic and steady-state behavior of SCs of different sizes and, also, have evaluated the impact of thermal and electrical stresses on the SCs' life. The majority of the SCs models can be grouped in two main categories. The first one is characterized by a time-domain parameters identification leading to a RC equivalent circuit model [1-4]. The second one is characterized by a parameter identification in the frequency-domain by using the electrochemical impedance spectroscopy (EIS) [5-9]. The performances and accuracy of these categories of modes are different. In particular, the first one cannot accurately represent the $\mathrm{SC}$ behavior during very slow charging/discharging cycles. On the other hands, the other category allows for obtaining more accurate SC dynamic models but requires high-end measurement systems.

Among all these models, there is a common drawback concerning the correct representation of the so-called redistribution phenomenon taking place during the SC charging and rest phases. As illustrated in the next section, this phenomenon is governed by the residual charge, $Q_{R}$, stored inside the SC when it is not connected to any circuit. From this standpoint, this paper aims at providing several experimental evidences of the SC behavior during dynamic cycles and aims at providing a specific $\mathrm{SC}$ model based on the one proposed in $[1,2]$.

The structure of the paper is the following: Section II discusses the experimental behaviors of SCs during subsequent charging-discharging cycles, Section III describes the proposed SC dynamic model and Section IV reports two examples for validating the proposed SC model. Section V finally analyses the effects of temperature on the evolution of the SC voltage profiles.

\section{EXPERIMENTAL CHARACTERIZATION OF SC DURING SUBSEQUENT CYLES}

Some previous works have already focused on the effect of the initial SC open-circuit voltage on the so called selfdischarge phenomenon [14-15]. These contributions have focused their study on the measurements, and relevant physical description, of the mechanisms driving the SC selfdischarge. They detected two main phases associated to the first hour and to a longer time window. In particular, they have described the SC charge movement through the electrode-electrolyte interface and how such as mechanism 
depends on the SC initial voltage. Nerveless none of these works accounted these mechanisms during the SC charging phase but only during the relaxation phase. In this respect, this section aims at experimentally investigate the effects of the residual charge stored in the SC during charging and discharging phases together with a possible way to properly model those effects.

\section{A. Effect of the SC residual charge during, and after, its charging phase}

The analysis of the SC behavior refers to experimental observations done on a specific 360-F device (although, in view of the similarity among SCs, these observation can be straightforward extended to other devices). In particular, subsequent identical cycles, composed by charging, discharging and relaxation phases, are applied to the $\mathrm{SC}$ to observe the effects of the SC residual charge during its charging phase. The procedure is described here below.

Before the tests, the targeted SC has been completely discharged (namely, short-circuited for 3 days) and, then, starting from an open circuit voltage $V_{O C}$ equal to zero, it has been charged with the rated current value of $3.6 \mathrm{~A}$ until its nominal voltage has reached $2.7 \mathrm{~V}$ (see Figure 1). This first charging takes $550 \mathrm{~s}\left(t_{1}\right.$ in Figure 1). Then, the SC has been discharged with a constant resistance until a minimum voltage value of $50 \mathrm{mV}$.

It is worth observing that, after the first $\mathrm{SC}$ cycle, it is hard to reach lower SC voltages because, during the $\mathrm{SC}$ charging and discharging, the diffusion phenomenon associated to charges migration toward the SC meso-micro pores electrodes, takes place. From a $V_{O C}$ value equals to 50 $\mathrm{mV}$, another charging phase with the same rated current has been performed. The time to reach the same final voltage (i.e $2.7 \mathrm{~V})$ was lower than $t_{1}(500 \mathrm{~s})$. The $\mathrm{SC}$ has been discharged and charged other six times with the same procedure describe above and, finally, the charging time reached an asymptotic constant value $t_{F}=472 \mathrm{~s}$.

As anticipated above, this behavior is associated to the presence inside the $\mathrm{SC}$ of a residual charge, $Q_{R}$, which participates during the subsequent $\mathrm{SC}$ usage. The effect of $Q_{R}$ is to reduce considerably the charging time compared to the value obtained when the SC has completely discharged (in our tests, such a difference is of $80 \mathrm{~s}$ ). Figure 1 summarizes what above illustrated. In particular, it shows the SC voltage profiles during subsequent charging phases (each curve refers to a charge subsequent to a discharge that have lasted to reach a $\mathrm{SC}$ voltage of $50 \mathrm{mV}$ ).

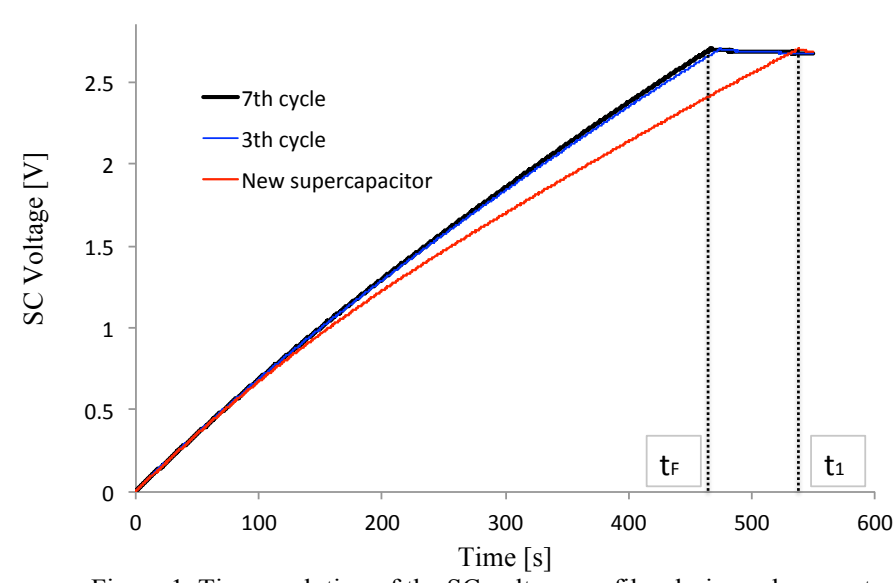

Figure 1. Time evolution of the SC voltage profiles during subsequent charging phases.

\section{B. Effect of residual charge during the $S C$ redistribution phase}

As know, the SC redistribution phenomenon is the process of homogenization of the charges on the whole SC electrode surfaces and it takes long time to be completed (typical time constants are the order of $700-800 \mathrm{~s}$ ). It is well known that the redistribution phenomenon is caused by the irregularity of the porous carbon structure of the SC electrodes involving a gradient of ion concentration within the SC electrolyte. This gradient depends on the different time constants of the macro, meso and micro pores of the interface electrodes-electrolyte [13]. Additionally, the presence of $Q_{R}$ during the relaxation phase increases the amount of charges participating to this phenomenon. The two main effects are: (i) reduction of the time needed for reaching the correspondent $V_{O C}$ (that involves lower times to complete the charges redistribution), (ii) increase the $V_{O C}$ value. Figure 2 illustrates this second main effect of the $Q_{R}$.

These two experimental observations are two different indicators of the same phenomenon, namely the gradient of charge within the SC electrolyte. It should be noticed that the different voltage dynamic behavior shown in Figure 2, starts to be non-negligible after $150 \mathrm{~s}$ after the disconnection of the SC supplying circuit. In this respect, an accurate SC model should be able to predict the right value of the voltage across the SC during long rest periods as well (concerning this point, it is worth noting that none of the models currently available in the literature is able to properly reproduce such a SC behavior).

As it will be illustrated next, if these two effects are not considered, the final voltage, as well as the whole SC voltage profile during a dynamic cycle, can largely differ from the measured values. 


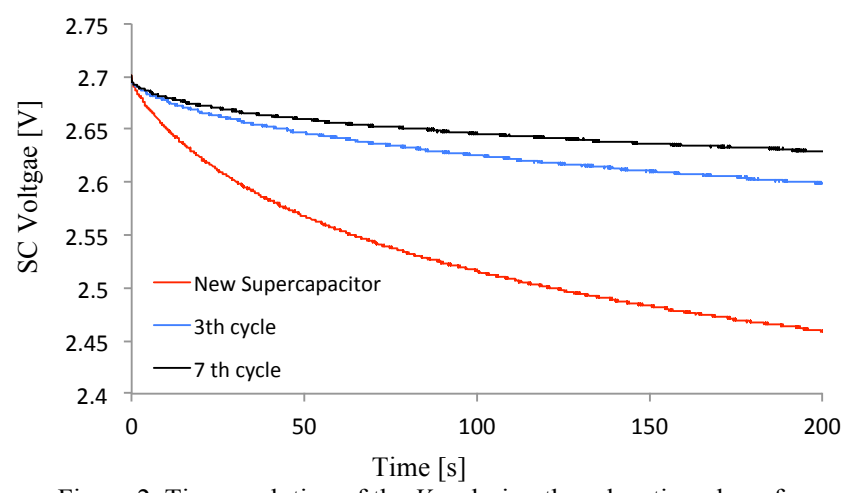

Figure 2. Time evolution of the $V_{S C}$ during the relaxation phase for different values of SC residual charge.

If the SC ageing process is neglected, it is possible to experimentally find the relationship between the final SC voltage and the corresponding open circuit voltage $V_{O C}$ (see Table I).

TABLE I. RELATION BETWEEN REACHED FINAL VOLTAGE $V_{F}$ AND $V_{O C}$

\begin{tabular}{|c|c|}
\hline & $V_{F C}(\mathrm{~V})$ \\
\hline 2.7 & 2.46 \\
\hline 2.4 & 2.13 \\
\hline 2.1 & 1.81 \\
\hline 1.8 & 1.49 \\
\hline 1.5 & 1.23 \\
\hline 1.2 & 0.97 \\
\hline 0.9 & 0.71 \\
\hline 0.6 & 0.48 \\
\hline 0.3 & 0.22 \\
\hline
\end{tabular}

\section{Evaluation of the residual charge}

Let consider a SC that is charged to reach a pre-defined $V_{F}$ and, subsequently, disconnected from the supplying circuit in order to reach its equilibrium (see Figure 3). For long time duration (around $3000 \mathrm{~s}$ ) the SC voltage will reach the open circuit voltage value $V_{O C}$. After the $\mathrm{SC}$ equilibrium, the device is discharged to a null value of the SC voltage and, therefore, we can associate to the $V_{O C}$ a relevant value of charge that, in view of what above-illustrated, is the $Q_{R}$. The phenomenon that can be observed is that during the $\mathrm{SC}$ charging we can associate an amount of charge, $Q_{C H}$, given to the $\mathrm{SC}$ in correspondence of the SC voltage equals to $V_{O C}$ (at time $t_{1}$ ). The difference between $Q_{R}$ and $Q_{C H}$, henceforth called $\Delta Q$, represents an amount of charge explaining the differences in the dynamic behavior of the targeted $\mathrm{SC}$ when it is cycled. This quantity $\Delta Q$ is used in the proposed $\mathrm{SC}$ model (see the definition of $I_{\mathrm{CH}}$ and $I_{\mathrm{RED}}$ in Figure 5). The procedure above described has been done in the whole voltage range from $50 \mathrm{mV}$ to $2.6 \mathrm{~V}$. In order to follow this procedure, the targeted SC should be completely discharged (i.e., short-circuited) for three days in order to obtain a starting $V_{O C}$ equal to zero. Since the improvement of the proposed model should be a tradeoff between accuracy and usability, it would be not convenient to totally discharge the $\mathrm{SC}$ before performing the measurements. In this respect, we have chosen to perform all the measurement with a starting $V_{O C}$ equal to $50 \mathrm{mV}$. From this stand point, considering the results shown in section II, this value of $V_{O C}$ involves a certain value of residual charge that has to be quantified. This value has been obtained by measuring the charge extracted from the SC during a three days short-circuit test that has started from an initial condition characterized by a $V_{O C}$ equal to $50 \mathrm{mV}$. This value has been added to the value of $\Delta Q$ calculated as result of the above procedure.

The non-linear link between $V_{O C}$ and $\Delta Q$ is illustrated in Figure 4.

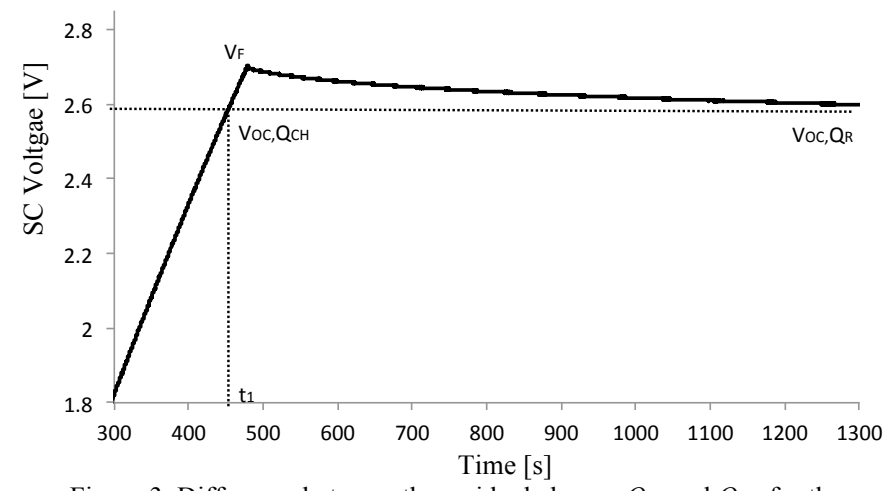

Figure 3. Difference between the residual charge, $Q_{R}$, and $Q_{C H}$ for the definition of $\Delta Q$ for a given $V_{O C}$ value.

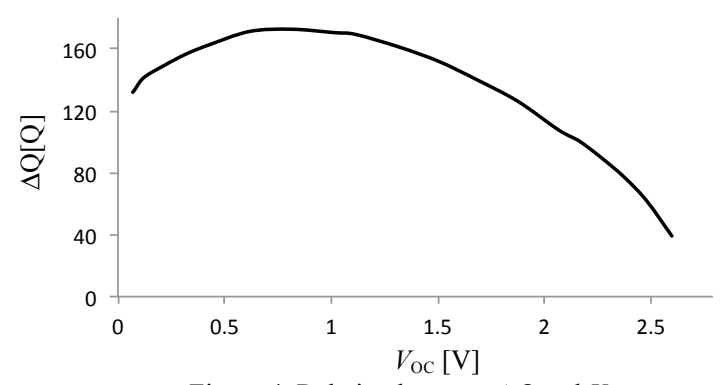

Figure 4. Relation between $\Delta Q$ and $V_{O C}$.

\section{PROPOSED SC MOdEL}

As anticipated in the introduction, the model proposed in this paper is an improved version of the one present in [1-2].

The main goal is to accurately predict the SC behavior when it is cycled with long time dynamics. Figure 5 illustrates the new proposed model, where: $R_{1}$ is the SC input electrode resistance; $R_{L}$ and $C_{V}$ are the resistance and the nonlinear capacitance of the so-called "SC network system model" (see [1-2]); $R_{2}$ and $C_{2}$ are the resistance and the nonlinear capacitance of the $\mathrm{SC}$ second branch, $R_{3}$ and $C_{3}$ are the resistance and the non-linear capacitance of the third SC branch. 
The evaluation of the parameters of the model presented in Figure 5 has been performed by applying a modified procedure of what proposed in $[1,2]$. In particular, the variation of the $\mathrm{SC}$ voltage has been divided into a certain number of intervals (in our case 10) between zero and the SC rated voltage (i.e., $0-2.7 \mathrm{~V}$ ). For each of these intervals a first set of the SC parameters has been evaluated by using the procedure given in [1-2]. Then, in order to improve the values of these parameters, a least square fitting procedure has been used.

The contribution of the paper concerning the SC model refers, as it can be observed form Figure 5, to the inclusion of two current sources $I_{\mathrm{CH}}$ and $I_{\mathrm{RED}}$. These two 'virtual' sources allow for improving the SC dynamics by taking into account respectively the diffusion of the residual charge during short phenomenon (i.e., charging/discharging phases) as well as during long time phenomenon (i.e., relaxation phases).

The determination of the parameters of these two current sources is described in what follows.

The virtual current generator $I_{\mathrm{CH}}$ is active only during charging and discharging phases. Since the redistribution phenomenon related to charging/discharging phases is mainly modeled by the so-called system network model composed by a RC circuit (see Figure 5), the current delivered by this generator can be represented by using an exponential function:

$i_{C H}(t)= \pm \frac{\Delta Q}{\tau} e^{-\frac{t}{\tau}}$

where $\tau$ is the time constant of the redistribution of $\Delta Q$. The value of this time constant depends on the size of the targeted $\mathrm{SC}$ (indeed, it depends on the macro-meso-micro structure of the electrodes-electrolyte interface) and, therefore, has been experimentally determined to be $1000 \mathrm{~s}$. The current will be positive if we are considering a charging phase, vice versa will be negative.

The other virtual generator $I_{\mathrm{RED}}$ is active only during relaxation phases. Previous works [14-15] have already experimentally demonstrated that the time evolution of the relaxation phase is pseudo-exponential and it can be mainly modeled by two $R C$ branches. The second branch takes into account the diffusion phenomenon of the charges though the medium sizes pores of the electrodes-electrolyte interface and its time constant is $\tau_{2}$. The third branch should take into account the same phenomenon though the micro sizes pores of the electrodes-electrolyte interface and its time constant is $\tau_{3}$. This behavioral law involves an exponential time evolution of the redistribution of the residual charge during relaxation phases. In this respect, the $\triangle Q_{R E D}$ should be delivered by following these two time constants associated to these two different dynamics of the relaxation phase. Consequently the current delivered by this generator can be described by the following equation:
$i_{R E D}(t)= \pm\left(\eta_{2} \frac{\Delta Q_{R E D}}{\tau_{2}} e^{-\frac{t}{\tau_{2}}}+\eta_{3} \frac{\Delta Q_{R E D}}{\tau_{3}} e^{-\frac{t}{\tau_{3}}}\right)$

where $\Delta Q_{R E D}$ is the amount of the residual charge available for the redistribution phase depending on the $\Delta Q$ quota used in the previous charging phase. The value $\Delta Q_{R E D}$ can be analytically calculated as:

$\Delta Q_{R E D}=\Delta Q-\int_{0}^{t_{c h}} i_{C H}(t) d t$

where $t_{c h}$ is the duration of the charging phase.

The parameters $\eta_{2}$ and $\eta_{3}$ are respectively the quota of $\Delta Q_{R E D}$ to be redistributed with the time constant $\tau_{2}$ and $\tau_{3}$. These two quantities, $\eta_{2}$ and $\eta_{3}$, have been experimentally evaluated. In particular, the amount of charges extracted during 24 hours has been evaluated by the time integral of the measured discharging current. Then, since the time evolution of the extracted charges is pseudo-exponential, a mathematical fitting provided by the following equation has been performed:

$q_{e x t}(t)=\eta_{2} \mathrm{e}^{-\tau_{2} t}+\eta_{3} \mathrm{e}^{-\tau_{3} t}$

At the end of the process the values of constants $\eta_{2}$ and $\eta_{3}$ are 0.3 and 0.7 respectively. The current of equation (2) will be positive if we are considering a redistribution phase just after a charging phase, it will be negative if we are considering a redistribution phase just after a discharging phase.

It is worth noting that the input of the proposed model are only: (i) the value of the open circuit voltage before any utilization of the targeted SC and (ii) the input current provided by a voltage or current source. Therefore, the proposed model is valid irrespective of the type of adopted source/load applied to the SC.

Additionally, it is important to note that these generators, $I_{\mathrm{CH}}$ and $I_{\mathrm{RED}}$, do not violate the charge balance of the SC since they are not injecting an external current but they model the movement of the charges already stored in the electrodeelectrolyte interface pores. The time-integral of currents provided by the two virtual current generators has been verified to not be higher than $\Delta Q$.

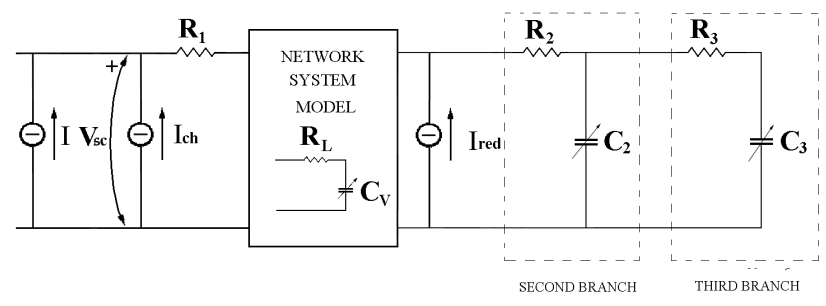

Figure 5. Proposed SC model.

A final comparison between the model here proposed and the Zubieta's one [1,2] concerns the time needed for the determination of the corresponding parameters. In this 
respect, the Zubieta's model requires one day of experiments (i.e., ten charging-discharging cycles). On the contrary, the model here proposed requires around two weeks of measurements in view of the need of inferring the time constants of the SC charge redistribution. The results and the improvements achieved with the model here proposed in section IV justifies the time needed for the determination of the parameters.

\section{EXPERIMENTAL VALIDATION OF THE PROPOSED MODEL}

Figure 6 and Figure 7 illustrate the comparison among the results coming from the measurement of a dynamic cycle composed by several charging, discharging and relaxation phases. The simulation results coming from the model presented in [1-2], the ones coming from a first optimized version of the model presented in [1-2] and the ones of the proposed model are shown in Figure 6 and Figure 7. In this respect, Figure 6 shows the results for a SC cycle with a low value of the charging current of $1.5 \mathrm{~A}$. This value of current is different from that used to determine the SC parameters, namely, $2 \mathrm{~A}$. This characteristic represents a real challenge on the dynamic modeling of SC. Figure 7 refers to a cycle with repetitive charging/discharging/redistribution phases. This specific cycle has been chosen since SCs are often subjected to these types of operations. The value of the charging current is equal to $2.3 \mathrm{~A}$

As it can be observed from Figures 6 and 7, the model derived from [1-2], that does not fully take into account the redistribution of the residual charge, does not appear able to correctly follow the dynamic behavior of the targeted SC. The relevant mean squared error is equal to $15 \cdot 10^{-3}$. The model here proposed, able to take into account the diffusion phenomenon of the difference in residual charge during charging/discharging phase, exhibits a better accuracy that results into a mean squared error of $6.8 \cdot 10^{-4}$. It is worth noting that such an error is one order of magnitude lower of the one of the model proposed in [1-2].

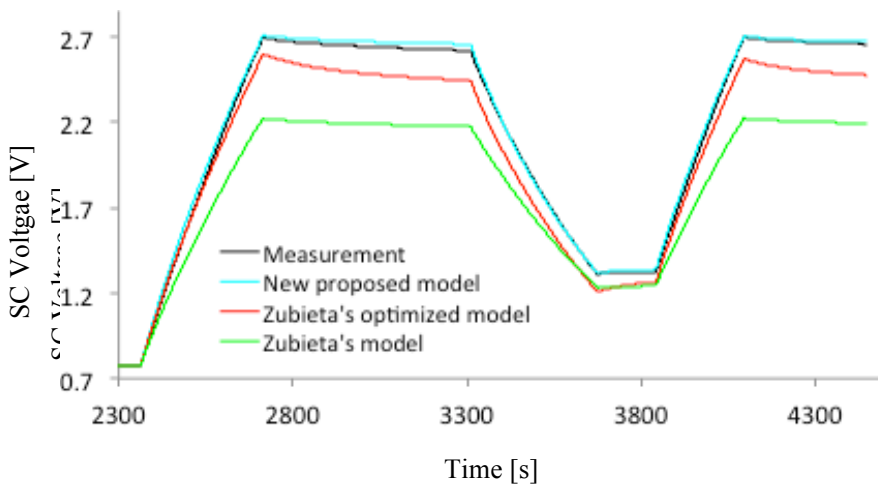

Figure 6. Comparison between experimental and simulation results.

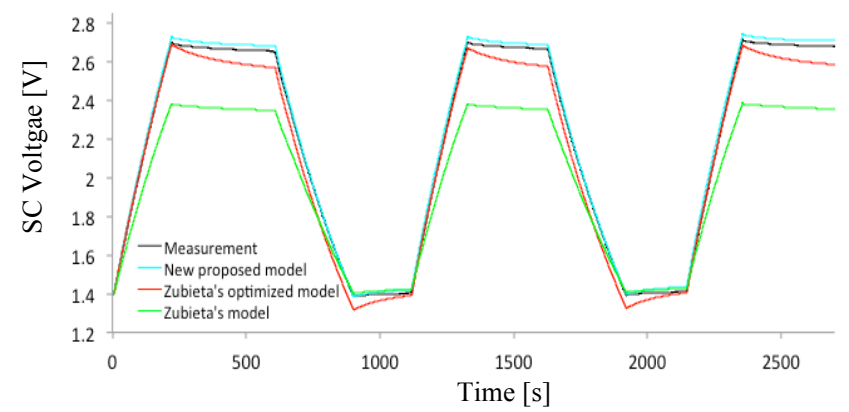

Figure 7. Comparison between experimental and simulation results.

\section{TEMPERATURE EFFECTS}

All the obtained measurements and associated parameters estimation have been performed in a climatic chamber at $25^{\circ} \mathrm{C}$ constant temperature. In this respect, this section investigates the variation of the $\mathrm{SC}$ voltage profiles related to environment temperature changes. As the SC dynamic model here presented is inferred from physical-experimental observations, we propose to apply the same approach to determine the temperature dependency of our model. In this respect, the same experimental results already presented in Section II, have been re-obtained at different temperatures. Figures 8 to 11 illustrate the time evolution of the SC voltage profile during subsequent charging phase at different temperatures, namely: $25^{\circ} \mathrm{C}, 45^{\circ} \mathrm{C}$ and $55^{\circ} \mathrm{C}$.

Actually, in order to not influence the SC lifetime, the selected $V_{F}$ value for these tests should be a tradeoff between stored energy and SC expected lifetime. In this respect the tests have been performed with a charging current equals $10 \mathrm{~A}$ and a $V_{F}$ of $2.45 \mathrm{~V}$.

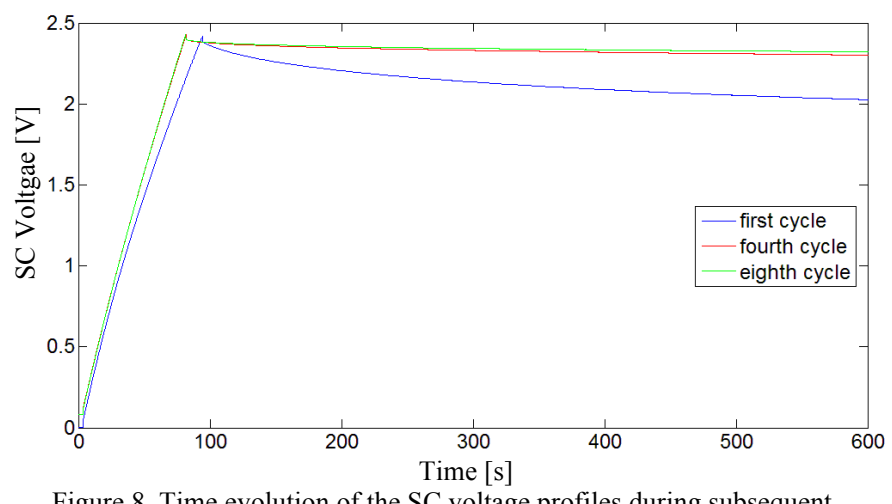

Figure 8 . Time evolution of the SC voltage profiles during subsequent charging phases at $25^{\circ} \mathrm{C}$. 


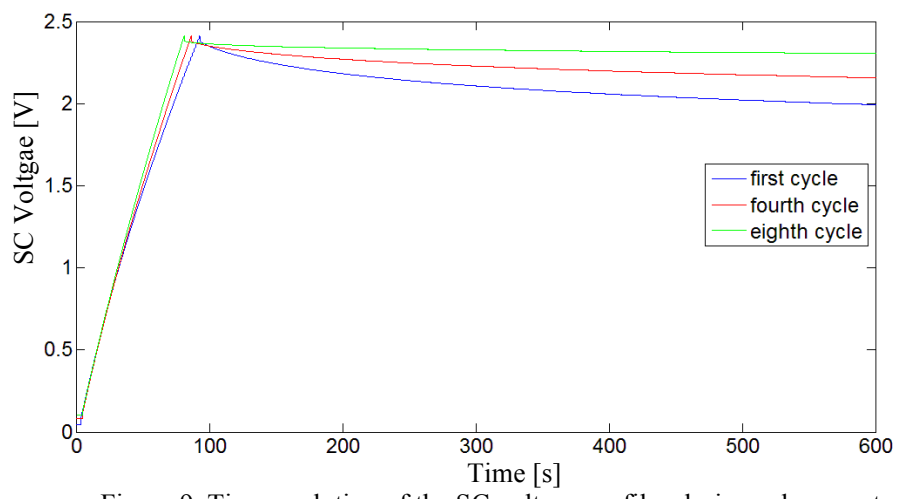

Figure 9. Time evolution of the SC voltage profiles during subsequent charging phases at $45^{\circ} \mathrm{C}$.

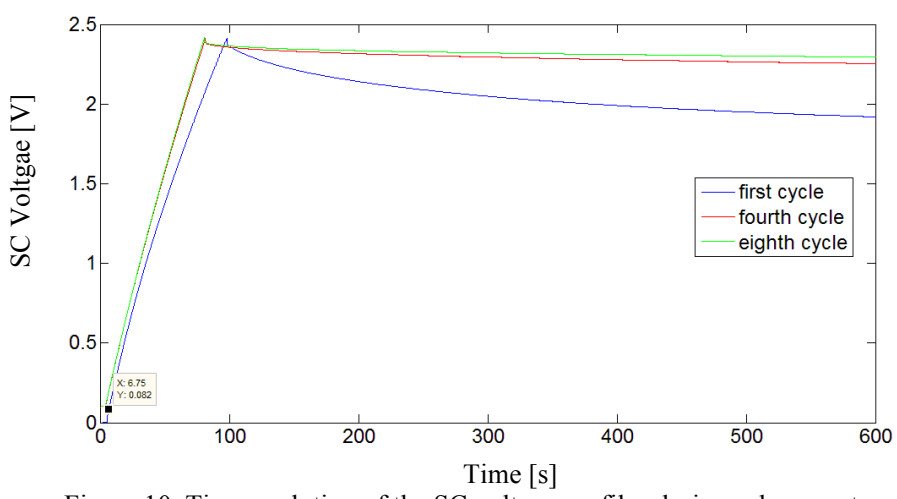

Figure 10. Time evolution of the SC voltage profiles during subsequent charging phases at $55{ }^{\circ} \mathrm{C}$.

Figure 11 illustrates the time evolution of the $V_{S C}$ during the relaxation phase at different considered temperatures.

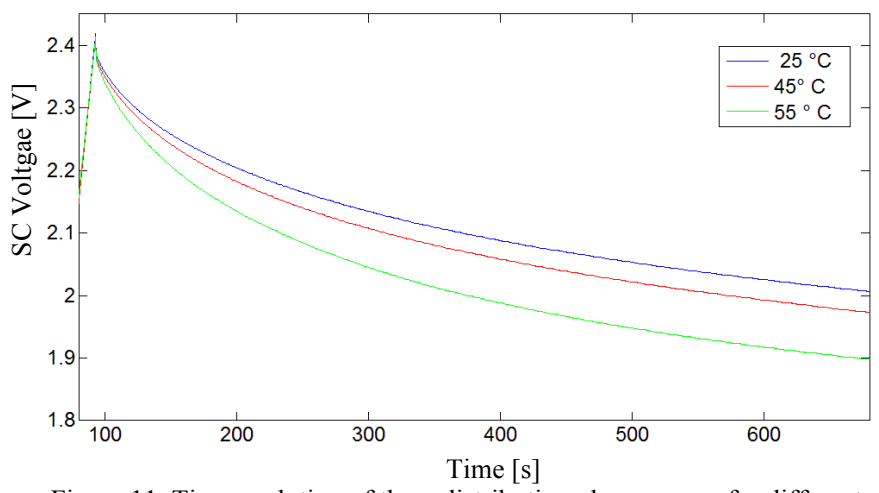

Figure 11. Time evolution of the redistribution phenomenon for different values of residual charge.

The main results of this last investigation are: (i) the higher the temperature the higher the time to reach the same $V_{F}\left(\right.$ at $55^{\circ} \mathrm{C}$ we can observe an increase in the charging time of $30 \%$ compared to the value at $25^{\circ} \mathrm{C}$ ); (ii) the time decay of the voltage during the relaxation phase increases as the $\mathrm{SC}$ temperature increase $\left(\right.$ at $55^{\circ} \mathrm{C}$ we can observe a difference in the $V_{S C}$ value equal to $8 \%$ compared to the value at $25^{\circ} \mathrm{C}$ ). A possible physical explanation of the above results can be related to the value of the residual charges. Indeed, at higher temperatures the redistribution phenomena of the charges stored inside the $\mathrm{SC}$ is less important during both the charging and the relaxation phases. This involves a lower value of residual charges along with a lower value of $\Delta Q$. These observations suggest a possible temperature adjustment of the SC dynamic model here proposed. Future work will be based on the experimental characterization of the relation between $\Delta Q$ and $V_{O C}$ for different temperatures (see Figure 4) and a consequent improvement of the dynamic model with a temperature dependency.

\section{CONCLUSIONS}

Starting from experimental evidences of the dynamic behavior of SCs, this paper has provided the basis for the development of an accurate SC model aiming at correctly represent the diffusion phenomenon of the $\mathrm{SC}$ residual charge. In particular, the model here proposed is based on experimental investigations of long relaxation phases and cycling of the targeted SC. Together with the model description, the paper has provided the experimental procedure required to determine the relevant model parameters.

The model has been experimentally validated with two different cycles characterized by different dynamic profiles. Finally, the paper has illustrated the influence of the temperature on the $\mathrm{SC}$ voltage profiles that are used to determine the parameters of the proposed SC model. The results have shown that there might be a large dependency between the SC residual charge and the temperature that deserves specific investigations.

\section{ACKNOWLEDGEMENTS}

The Authors gratefully acknowledge the EOS-Holding and the Swiss Innovation and Technology Committee (CTI) as the results shown in this paper have been partially obtained within ongoing research activities sponsored by these entities.

\section{REFERENCES}

[1] F. Belhachemi, S. Rael, B. Davat, "A physical based model of power electric double-layer supercapacitors," IEEE Conference Record of Industry Applications Conference. 2000, vol.5, pp.3069-3076, 2000.

[2] L. Zubieta, R. Bonert, "Characterization of double-layer capacitors for power electronics applications," IEEE Transactions on Industry Applications, vol.36, Issue.1, pp.199-205, 2000.

[3] N. Bertrand, J. Sabatier, O. Briat, J. Vinassa, "Embedded Fractional Nonlinear Supercapacitor Model and Its Parametric Estimation Method," IEEE Transactions on Industrial Electronics, vol.57, Issue.12, pp.3991-4000, 2010.

[4] A.S. Weddell, G.V. Merrett, T.J. Kazmierski, B.M. Al-Hashimi, "Accurate Supercapacitor Modeling for Energy Harvesting Wireless Sensor Nodes," Circuits and Systems II: Express Briefs, vol.58, Issue.12, pp.911-915, 2011.

[5] V. Musolino, L. Piegari, E. Tironi, "New full frequency range supercapacitor model with easy identification procedure," IEEE Transactions on Industrial Electronics, vol.60, Issue. 1, pp.112-120, 2011.

[6] F. Rafik, H. Gualous, R. Gallay, A. Crausaz, A. Berthon, "Frequency, thermal and voltage supercapacitor characterization and modeling", Journal of Power Sources, vol.165, Issue. 2, pp.928-934, 2007.

[7] N. Rizoug, P. Bartholomeus, P. Le Moigne, "Modeling and Characterizing Supercapacitors Using an Online Method," IEEE Transactions on Industrial Electronics, vol.57, Issue.12, pp.3980-3990, 2010.

[8] S. Buller, E. Karden, D. Kok, R.W. De Doncker, "Modeling the dynamic behavior of supercapacitors using impedance spectroscopy," 
IEEE Transactions on Industry Applications, vol.38, Issue.6, pp.16221626, 2002.

[9] Sang-Hyun Kim, Woojin Choi, Kyo-Bum Lee, Sewan Choi, "Advanced Dynamic Simulation of Supercapacitors Considering Parameter Variation and Self-Discharge," IEEE Transactions on Power Electronics, , vol.26, Issue.11, pp.3377-3385, 2011.

[10] A. Banaei, A. Khoobroo, B. Fahimi "Online Estimation of State of Charge in Li-Ion Batteries Using Impulse Response Concept," Smart Grid, IEEE Transactions on , vol.3, no.1, pp.360-367, March 2012

[11] El Brouji, E.-H.; Briat, O.; Vinassa, J.-M.; Bertrand, N.; Woirgard, E.; , "Impact of Calendar Life and Cycling Ageing on Supercapacitor Performance," Vehicular Technology, IEEE Transactions on , vol.58, no.8, pp.3917-3929, Oct. 2009.

[12] O. Bohlen, J. Kowal, and D.-U. Sauer, "Ageing behaviour of electrochemical double layer capacitors: Part I. Experimental study and ageing model," J. Power Sources, vol. 172, no. 1, pp. 468-475, Oct. 2007.

[13] O. Bohlen, J. Kowal, and D.-U. Sauer, "Ageing behaviour of electrochemical double layer capacitors: Part II. Lifetime simulation model for dynamic applications,"J. Power Sources, vol. 173, no. 1, pp. 626-632,Nov. 2007.

[14] Y, Diab, P. Venet, H. Gualous, G. Rojat; "Self-Discharge Characterization and Modeling of Electrochemical Capacitor Used for Power Electronics Applications," Transactions on Power Electronics, IEEE, vol.24, no.2, pp.510-517, Feb. 2009.

[15] B W Ricketts, C Ton-That, «Self-discharge of carbon-based supercapacitors with organic electrolytes » in Journal of Power Sources Vol. 89, 2000, pp. 64-69.

[16] K. Maximilina, J. Kowal, D. U. Sauer, "Modelling the effects of charge redistribution during self-discharge of supercapacitors", Electrochimica Acta n.55, pp.7516-7523, 2010. 